\title{
Caracterización fractal de angioresonancia magnética cerebral
}

\author{
Guillermo De la Rosa-Orea ${ }^{1}$, Alfonso Medel-Rojas ${ }^{1}$, Tomás Morales-Acoltzi ${ }^{2}$, Enrique \\ Martínez Ramírez ${ }^{1}$, Juan Manuel López-Oglesby ${ }^{1}$, Manuel González-Pérez ${ }^{1}$ \\ ${ }^{1}$ Centro Interdisciplinario Posgrados, Ingeniería Bio-médica UPAEP, Puebla, México \\ gdelarosa7@gmail.com, alfonso.medel.r@gmail.com,e_martinez_r@hotmail.com, \\ \{juanmanuel.lopez, manuel.gonzalez\}@upaep.mx \\ ${ }^{2}$ Centro de Ciencias de la Atmósfera, UNAM, México \\ acoltzi@atmosfera.unam.mx
}

\begin{abstract}
Resumen. Aplicamos conceptos de la geometría fractal (GF) y procesamiento digital de imágenes (PDI) para caracterizar imágenes médicas de angioresonancia magnética cerebral (ARM) de pacientes normales y otros que presentan la patología de disminución del calibre arterial, con reducción de la luz interior de arterias. La caracterización de imágenes ARM permite obtener conocimiento para desarrollar metodologías alternativas para dotar de inteligencia a herramientas de apoyo al diagnóstico médico. Se efectuó un postprocesamiento a las imágenes de ARM y se aplicó el método de "Box-Counting" para obtener su dimensión fractal (DF). Se desarrolló con MATLAB V10 un análisis de la capacidad de ocupar espacio de acuerdo a la GF. Los resultados muestran que la metodología implementada, permite identificar la patología de manera no supervisada y en tiempo real, apoyando la clasificación del triage médico del paciente, en forma operacional, solicitando así la intervención inmediata de un experto clínico, para su adecuada canalización.
\end{abstract}

Palabras claves. Angio Resonancia de Cerebro, Geometría Fractal, Box Counting, Dimensión Fractal, Procesamiento de Imágenes.

\section{$1 \quad$ Introducción}

La angioresonancia magnética o angiografía por resonancia magnética (ARM) es una aplicación de la resonancia magnética nuclear (RMN) para la visualización de la vasculatura del cuerpo humano. En particular para esta investigación nos enfocamos a la 
región cerebral. Las imágenes proporcionadas por el escaneo de un estudio de ARM cerebral, incluye, por lo general, cortes en los 3 planos anatómicos, coronal, sagital, y transversal, para que de esta manera se obtenga una visión completa de la circulación arterial cerebral, Figura 1. Algunas de las patologías asociadas a la vasculatura cerebral entre otras, incluyen el aneurisma, que consiste en la dilatación de un vaso sanguíneo, malformaciones arteriovenosas que ocurre en donde no hay lecho capilar y hay paso directo de sangre del lado arterial al lado venoso, otra de las patologías que reportan los médicos radiólogos, es la disminución del calibre arterial, Figura 2, es decir, se reduce la luz interior de las arterias; los vasos sanguíneos estrechos dificultan y, a veces, bloquean el flujo de sangre, y si el flujo de sangre se detiene, los órganos que depende de éste se pueden dañar o morir, también el bloqueo de las arterias del cerebro pueden llevar a un ataque de apoplejía, que causa parálisis contralateral a la región del cerebro en donde está ocurriendo una isquemia cerebral o una hemorragia cerebral $[8,9]$. Una vez identificada la patología, el paciente debe ser clasificado de acuerdo al triage hospitalario, o bien; el médico tratante define el tratamiento a aplicar [12].

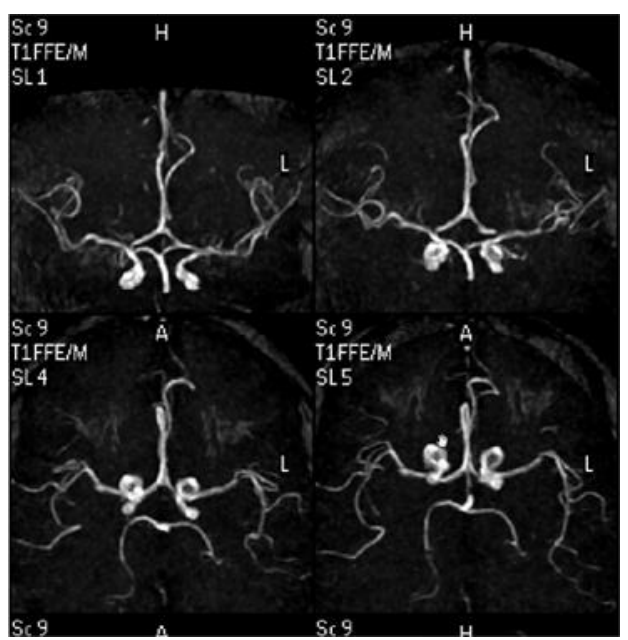

Fig. 1. Cuatro cortes de ARM cerebral de paciente normal.

Aplicar nuevas metodologías de PDI para identificar patologías presentes en las imágenes médicas nos permite avanzar en la tarea del diagnóstico automatizado y así, dotar a las herramientas de apoyo con cierta inteligencia. Las metodologías de post procesamiento actuales han llegado a ser bastante complejas, mejorar su desempeño implica agregarle más complejidad (7) y además, por lo general, en forma operacional, es necesario que dichos algoritmos se apliquen en tiempo real. Para lograr esto, debemos en principio reducir términos o ecuaciones; otra alternativa es buscar enfoques diferentes (4). 
La teoría del caos y la GF, nos plantean una manera alternativa de análisis, estas nuevas metodologías están dando resultados positivos a muchos de los procesos aparentemente complejos y caóticos presentes en la naturaleza [1], también gracias a la recursividad, han logrado reducir el costo computacional, de hecho, se está dando actualmente una tendencia mundial de aplicar este enfoque no lineal (5).

Los métodos tradicionales de PDI para segmentar y detectar bordes incluyen el trabajo con métodos principalmente lineales y que dependen de la derivada, como por ejemplo los métodos de Canny, Sobel, Prewitt y Roberts, Figura. 3. En esta línea el estado del arte ha llegado a explorar métodos bastante complejos y con formulaciones en base a modelación matemática [6]. Sin embargo, los resultados aún no son tan satisfactorios respecto a una utilidad médica confiable. Por lo que en este artículo aplicamos fundamentos de la GF a las imágenes médicas de ARM, para obtener una caracterización de dichos elementos y extraer información que ayude a generar conocimiento nuevo y útil aplicado en el desarrollo de herramientas de apoyo diagnóstico. La DF nos da una medida de esa complejidad y el valor del espacio ocupado de acuerdo a la TF.

Las bases de la GF se describen en la sección 2. El resto del documento se presenta de la siguiente manera: En la sección 3 se detallan la base de datos y la metodología pertinente. La sección 4 presenta los resultados de la caracterización y finalmente la sección 4 contiene un resumen y las conclusiones.

\section{Bases de la geometría fractal}

La GF, se desarrolló a partir de los estudios sobre la complejidad efectuados por Benoit Mandelbrot en las décadas de 1960 y 1970(1). Mandelbrot acuñó el término fractal del latín "fractus" (roto), para de esta manera resaltar la naturaleza fragmentaria e irregular de las formas. Los fractales presentan auto-semejanza, es decir, tienen el mismo aspecto tras ser sometido a un cambio de escala, cada pequeña porción es muy parecida a la estructura total. La auto-semejanza puede ser de dos tipos: exacta y estadística. Cuando se hace una ampliación de cierta región del objeto y ésta presenta una repetición exacta de sus detalles se dice que es auto-semejanza exacta, en cambio si los detalles del objeto no se repiten exactamente, en si lo que se repite en este caso son las propiedades estadísticas de dichos detalles. La mayor parte de las configuraciones de la naturaleza obedecen a la autosemejanza estadística. Una de las maneras de caracterizar a los fractales es por su dimensión, la DF, es un número que expresa su complejidad, no es un número entero, a diferencia de las dimensiones de las figuras geométricas elementales, es un número fraccionario. La GF tiene como caso especial a la geometría euclidiana (GE). La GE nos proporciona las herramientas necesarias para obtener las propiedades y medidas de elementos creados por el hombre tales como puntos, líneas, planos, volúmenes y los conjuntos creados por sus com-binaciones, de esta manera se obtienen modelos simplificados del objeto o del proceso en estudio. 
Sin embargo, las formas y los procesos de la naturaleza tales como los órganos del cuerpo humano, las nubes, las montañas, los árboles, los vegetales, los diferentes procesos y sistemas como el meteorológico no son fácilmente caracterizados por la $\mathbf{G E}$, en cambio la GF nos proporciona un modelo conceptual para describir los sistemas y objetos de la naturaleza que presentan esa forma irregular o interrum-pida.

La DF nos indica y mide ese grado de irregularidad e interrupción, en otras palabras, su capacidad de ocupar espacio que tiene el objeto. Ciertas curvas planas muy irregulares, sin llegar a ocupar las dos dimensiones, sin embargo, quedan descritas por la DF que está entre 1 y 2, o bien para ciertas superficies llenas de convoluciones la DF es intermedia entre 2 y 3 ; también sobre la recta se pueden encontrar valores de la DF entre 0 y 1 . El método más utilizado para calcular la DF es conocido como "Box Counting" el cual se describe en la sección 3.

\section{Datos y metodología}

Una imagen de resonancia magnética, IRM, o imagen por resonancia magnética nuclear (NMRI, por sus siglas en inglés "Nuclear Magnetic Resonance Imaging") es una técnica no invasiva, sin radiación que utiliza el proceso de la resonancia magnéti-ca y la radio frecuencia para obtener información sobre la estructura y composición del interior del cuerpo humano.

Esta información es procesada por computadoras y transformada en imágenes. Éstas son utilizadas, principalmente, para observar alteraciones y anomalías en los diferentes tejidos, lo que permite detectar patologías.

Se obtuvieron imágenes de ARM, cerebral, que presentan menor complejidad ya que solamente muestran la vasculatura cerebral. Se seleccionaron imágenes de pacientes normales, Figura 1 y de pacientes que presentan disminución del calibre en las arterias cerebrales, Figura 2. Mediante la DF obtenemos la cantidad del espacio que ocupan las arterias, de hecho, lo vemos con un menor radio sobre 2 dimensiones.

El formato original de las imágenes de ARM se encuentran en escala de grises, es decir, una gama de tonalidades de gris que van del negro al blanco (0-255). Para calcular la DF es necesario, simplificar la imagen, por lo que se lleva a cabo los siguientes pasos: primero, se aplica el método del valor de umbral que nos permite segmentar las arterias del resto de la imagen; segundo, obtenemos las imágenes equivalentes binarias, en donde persisten dos valores, negro y blanco $(0,1)$, Figura 4 ; y tercero, aplicar el método de BoxCounting y así obtener el valor numérico de la DF.

En este caso los pixeles con valor 1 son los que contienen información. En esta investigación se tuvo acceso al mismo número de casos de pacientes sanos y con disminución del calibre arterial, con un total de 72 imágenes. 


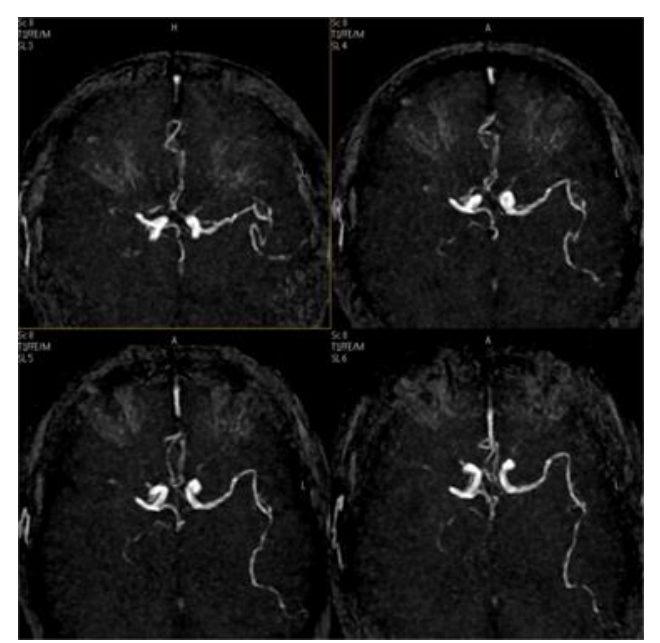

Fig. 2. Cuatro cortes de ARM cerebral de paciente con Disminución del calibre en la arteria cerebral media derecha.

\subsection{El método del valor umbral}

El método de valor de umbral pertenece a los más antiguos métodos del PDI. La finalidad de este algoritmo es segmentar imágenes, es decir, separar los objetos de una imagen que nos interesen del resto. En las situaciones más sencillas se puede decidir qué píxeles conforman los objetos que buscamos y qué píxeles son solo el fondo. Este método ha sido utilizado ampliamente en el reconocimiento de patrones (2).

Los métodos de segmentación asignan a cada píxel o voxel a un cierto grupo, llamado comúnmente segmento. La imagen está compuesta por valores numéricos (uno o más valores de color para cada píxel, RGB). La pertenencia de un píxel a un cierto segmento se decide mediante la comparación de su nivel de gris con un cierto valor umbral. El nivel de gris de un píxel equivale a su nivel de luminosidad; el resto de la información sobre el color no se tiene en cuenta.

El método del valor umbral binariza la imagen original, es decir, se construyen dos segmentos: el fondo de la imagen y los objetos buscados, las arterias. La asignación de un pixel a uno de los dos segmentos (0 y 1) se consigue comparando su nivel de gris g con un cierto valor umbral preestablecido $t$, "threshold", generalmente, se considera el valor promedio de tonos de gris, sin embargo en esta investigación las imágenes presentan mayor carga de tonos negros, lo que implica que el valor promedio no funcione, de tal forma que el experto neurólogo, selecciona el tono objetivo, y como la distribución de éste no es homogénea con respecto al patrón de las arterias, queda determinado después de al menos 
tres pruebas, intentado conservar el 100\% de las estructuras arteriales. El cálculo se realiza mediante:

$$
\mathrm{T}_{\text {global }}(\mathrm{g})=\{(0 \text { si } \mathrm{g}<\mathrm{t} \text { y } 1 \text { si } \mathrm{g} \geq \mathrm{t})\}
$$

Lo que nos indica que cada pixel pertenece, obligatoriamente, a un segmento y solo uno. La calidad de la segmentación depende de la imagen original por analizar.
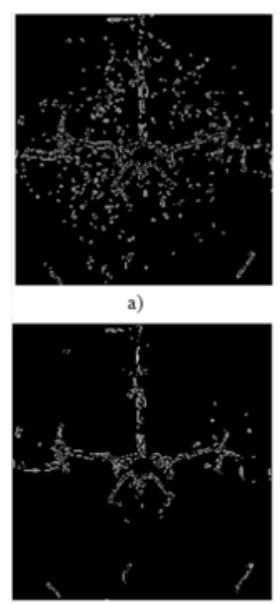

c)
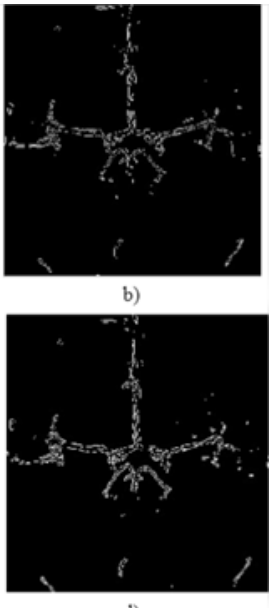

Fig. 3. Métodos tradicionales de segmentación (a) Canny, (b) Sobel, (c) Prewit, (d) Roberts.

\subsection{El método "Box Counting"}

Para el cálculo numérico de la DF se emplea el algoritmo conocido como "BoxCounting", o conteo de cajas, sí trabajamos en 3D o cuadrados en 2D, en su caso más general se trata con hipercubos. El método también, nos determina si la estructura en la imagen presenta fractalidad y auto-semejanza si el resultado es un no entero.

La metodología consiste en trazar una malla sobre la imagen formando cajas cuadradas de tamaño $\boldsymbol{r}$, figura 5. Lo que podemos observar es que algunas cajas van a contener arteria y otras van a estar vacías, es decir, cajas de lado $\boldsymbol{r}$ con $N(\boldsymbol{r})$ cajas ocupadas, el siguiente paso es cambiar el tamaño de $\boldsymbol{r}$, es decir, hacer el conteo de cajas ocupadas a diferentes escalas. La variación de escala, de un paso al siguiente, es dependiente del tamaño de la imagen y de los múltiplos de ese tamaño.

Se presentan los resultados de las mediciones en una gráfica log-log, Figura 6, en el eje horizontal mostramos el logaritmo del inverso del tamaño de lado de las cajas, puesto que, en principio, el proceso es ad-infinitum disminuyendo la longitud de medida, $\ln (1 / r)$ 
y en el eje vertical, el logaritmo del número de cajas ocupadas; $\ln (N(r))$; importándonos el comportamiento lineal de la gráfica mostrándonos la existencia de una ley de potencia.

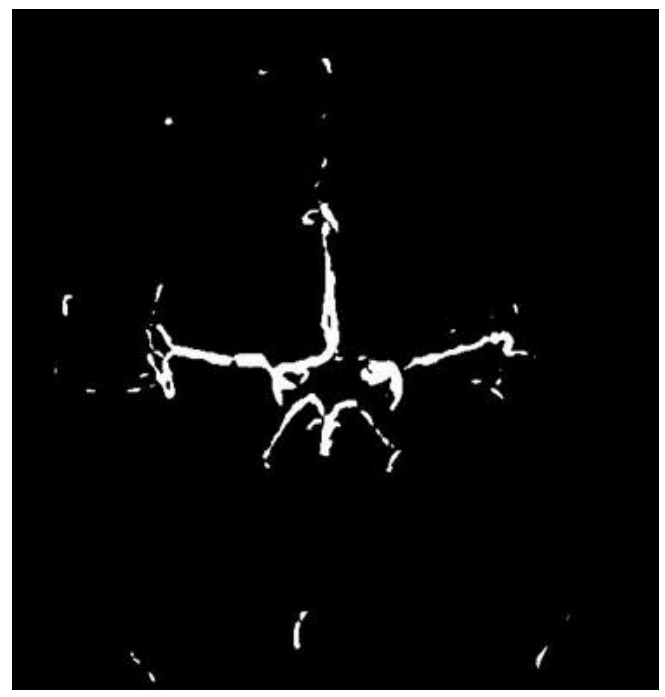

Fig. 4. ARM binarizada.
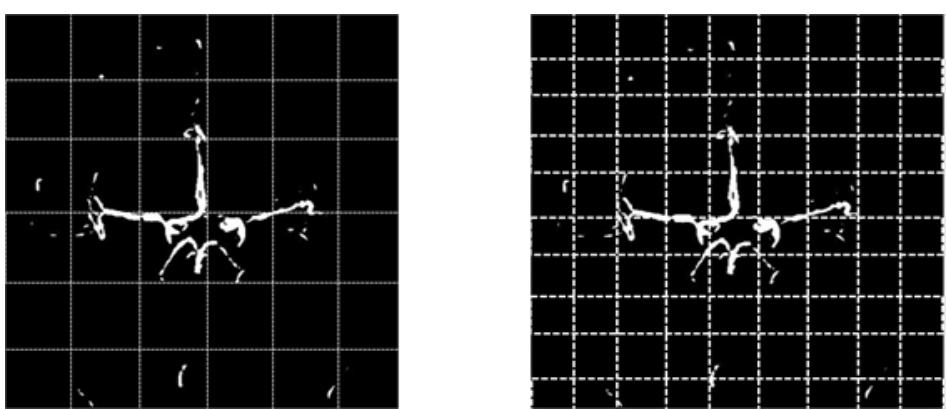

Fig. 5. Rejilla de recubrimiento para el método "box counting".

Los puntos aparecen a lo largo de una recta positiva, y se ajusta a la ecuación general de una línea recta.

$Y=m x+b$, con $\boldsymbol{b}$ la ordena al origen, $\boldsymbol{m}$ la inclinación de la recta, $\boldsymbol{x}$ la variable independiente e $\boldsymbol{y}$ la variable dependiente. 
Despejando a $\boldsymbol{D}$, tenemos:

$$
N(r)=C r^{-D}
$$

Que es una ley de potencia de exponente $-D$, despejando a $D$ determinamos la dimensión fractal de la estructura en estudio.

$$
D=\ln (1 / r) / \ln (N(r))
$$

\section{$4 \quad$ Resultados y conclusiones}

Uno de los objetivos principales de esta investigación, fue demostrar que la DF representa la capacidad de ocupar espacio de las estructuras contenidas en las imágenes de ARM, al encontrar valores generalmente no enteros.

La gráfica de barras de la Figura 7, muestra los resultados de los experimentos realizados, como se puede observar las barras de color rojo representan a pacientes con ARM normal, en color azul a pacientes con disminución del calibre arterial, con valores en general menores a 1. Está clara diferencia hace que el software pueda discriminar certeramente si la patología está presente o no.

De acuerdo a los resultados, podemos concluir que los valores calculados de la DF, nos permitieron caracterizar las imágenes de ARM. Se logró desarrollar un sistema en el lenguaje de MATLAB V10, para realizar los experimentos numéricos y evaluar los resultados obtenidos desde el punto de vista de la GF. El software sugiere, sin ningún otro tipo de apoyo, si la patología está o no en las imágenes.

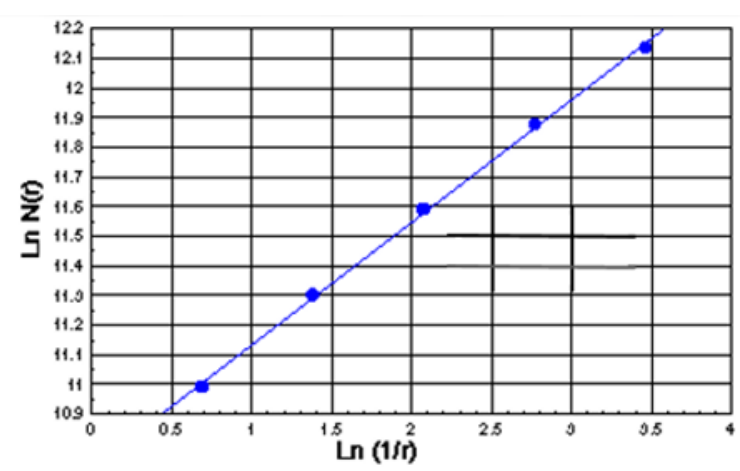

Fig. 6. Gráfica log-log. 

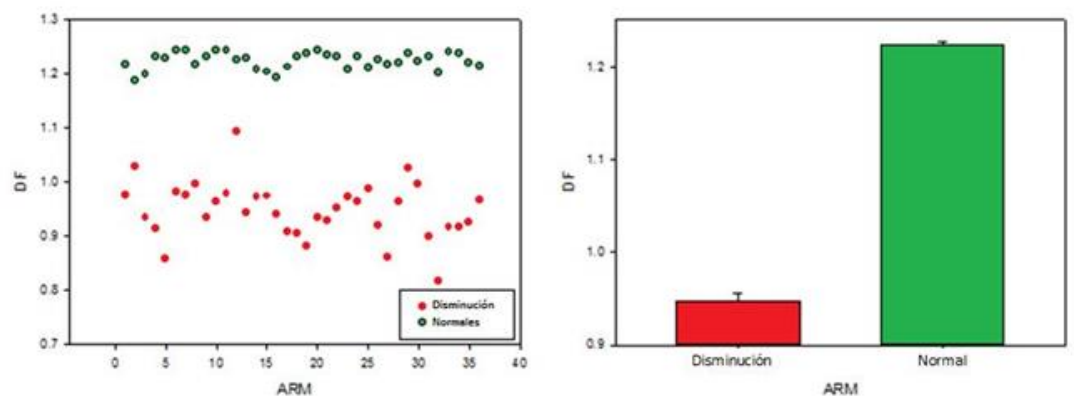

Fig. 7. Resultados, valores DF

El método aplicado en base a la GF nos permitió identificar pacientes con disminución del calibre arterial. Es importante resaltar que la interacción entre el experto neurólogo y el desarrollador del sistema fue de vital importancia, por lo que se abre una nueva área de oportunidad para sistematizar y automatizar el diagnóstico.

\section{$5 \quad$ Trabajos futuros}

Siguiendo con el enfoque no lineal, una de las tareas futuras, es aplicar los conceptos de la teoría multifractal. Muchas de las formas anatómicas contenidas en las imágenes médicas aparentemente no son auto-semejantes, sin embargo, éstas pueden verse como una mezcla o combinación de diferentes fractales [5].

\section{Referencias}

1. Mandelbrot, B. B.: The Fractal Geometry of Nature, W. H. Freeman and Co., New York, (1982)

2. Rafael C. González, Richard E. Woods.: Digital Image Processing. AddisonWesley, Reading Mass 1992. ISBN 0-201-50803-6 (1992)

3. Nobuyuki Otsu, A threshold selection method from grey level histograms. In: IEEE Transactions on Systems, Man, and Cybernetics. New York 9, S.6266.ISSN 1083-4419 (1979)

4. J. A. Tenreiro Machado, and I say to myself: what a fractional world. Fractional calculus \& applied analysis (2011)

5. Liebovitch S. Larry, Fractals and Chaos: Simplified for the Life Sciences, OXFORD University Press, pp 268, (1998) 
6. Xianghua Xie, Member, IEEE, and Majid Mirmehdi, Senior Member, IEEE: Magnetostatic Active Contour Model. IEEE Transactions on pattern analysis and machine intelligence, Vol. 30, No. 4, April (2008)

7. Jacques Lévy Véhel: Introduction to the Multifractal Analysis of Images In Fractal Image Encoding and Analysis (1998)

8. Torsten B. Moeller, Emil Reif: Pocket Atlas of Sectional Anatomy CT and MRI, Vol.1 head neck Spine And Joints, Thieme Stutgart New York (2000)

9. Anne G. Osborn: Diagnostic Imaging Brain, Second Edition, ISBN 0-7216-29059, (2004)

10. S. Sukumaran, Dr. M. Punithavalli: Retina Recognition Based on Fractal Dimension. IJCSNS International Journal of Computer Science and Network Security, VOL.9 No.10, October (2009)

11. R. Lopes, A. Ayache, N. Makni, P. Puech, A. Villers, S. Mordon and N. Betrouni: prostate cancer characterization on MR images using fractal features. 2011 American Association of Physicists in Medicine, (2011)

12. Instituto Mexicano del Seguro Social, http://www.imss.gob.mx 